\title{
Glaucoma de ângulo fechado secundário à inflamação orbitária inespecífica: relato de caso
}

\author{
Angle-closure glaucoma secondary to nonspecific orbital inflammatory: case report
}

\author{
Luciano Sólia Násser ${ }^{1}$ \\ Vera Lucia Liendo da Costa ${ }^{2}$ \\ Marcel Papa Taniguchi ${ }^{3}$ \\ Anamaria Bolanho ${ }^{4}$ \\ Ana Maria Noriega Petrilli ${ }^{5}$
}

Trabalho realizado no Centro de Estudos de Oftalmologia Dr. José Carlos Gouvêa Pacheco - Universidade de Mogi das Cruzes - UMC - Mogi das Cruzes (SP) - Brasil.

${ }^{1}$ Residente do Programa de Residência em Oftalmologia da Universidade de Mogi das Cruzes - UMC - Mogi das Cruzes (SP) - Brasil.

${ }^{2}$ Residente do Programa de Residência em Oftalmologia da UMC - Mogi das Cruzes (SP) - Brasil.

Residente do Programa de Residência em Oftalmologia da UMC - Mogi das Cruzes (SP) - Brasil.

${ }^{4}$ Médica Responsável pelo Setor de Glaucoma do Programa de Residência em Oftalmologia da UMC - Mogi das Cruzes (SP) - Brasil.

${ }_{5}^{5}$ Professora Titular da Disciplina de Oftalmologia e Coordenadora do Programa de Residência em Oftalmologia da UMC - Mogi das Cruzes (SP) - Brasil.

Endereço para correspondência: Rua Loureiro da Cruz, 35 - Apto. 508 - Aclimação - São Paulo (SP) CEP 01529-020

E-mail: lucianopcu@ig.com.br

Recebido para publicação em 06.09.2006

Última versão recebida em 20.07.2007

Aprovação em 06.08.2007

\section{RESUMO}

Inflamação orbital não-específica apresenta diversas formas clínicas. $\mathrm{O}$ envolvimento do segmento posterior do olho, geralmente, por contigüidade pode trazer sérios danos à função visual. A esclerite posterior, em geral, acarreta prejuízo permanente da visão e raramente evolui com glaucoma agudo. Relato do caso: E.N., 24 anos, masculino, negro apresentando queixa de dor em OE há dez dias, acompanhada de diminuição da acuidade visual, mal-estar geral, náuseas e vômitos. Ao exame oftalmológico apresentava proptose, restrição da movimentação e edema na pálpebra superior de OE. AV c/c: 20/20 e CD 1,5m. À biomicroscopia, apresentava em OE hiperemia conjuntival, córnea com precipitados endoteliais, câmara anterior rasa, células e "flare" na câmara anterior $2+$. Pressão intra-ocular (Po) de $14 \mathrm{mmHg}$ em OD e $34 \mathrm{mmHg}$ em OE. A gonioscopia em $\mathrm{OE}$ evidenciava ângulo fechado $360^{\circ}$, não se visualizando linha de Schwalbe. O mapeamento de retina revelava aumento da tortuosidade vascular e edema do pólo posterior. O tratamento para o glaucoma agudo foi instituído, ainda em ambiente hospitalar, e solicitados exames complementares. O exame de ultra-som ocular e TC de órbita revelaram espessamento difuso da parede ocular e da musculatura extrínseca ocular. Os demais exames apresentaram-se dentro da normalidade. A hipótese diagnóstica foi de inflamação orbitária anterior nãoespecífica aguda com envolvimento do segmento posterior do globo ocular, complicado por glaucoma agudo. Instituiu-se tratamento com prednisona $60 \mathrm{mg} /$ dia via oral. Após duas semanas do início da corticoterapia sistêmica, apresentava-se assintomático com nítida regressão da proptose, do quadro de esclerite e normalização da Po $(11 \mathrm{mmHg} \mathrm{em}$ $\mathrm{AO})$. O presente caso, apesar de pouco freqüente, mostra que o glaucoma agudo pode estar presente em um quadro inflamatório orbitário e deve ser tratado com corticoterapia sistêmica, além da medicação tópica.

Descritores: Doenças orbitárias/complicações; Esclerite; Glaucoma de ângulo fechado/ etiologia; Relatos de casos [Tipo de publicação]

\section{INTRODUÇÃO}

Inflamação orbital não-específica, antigamente chamada de pseudotumor, se manifesta sob a forma de uma inflamação aguda ou subaguda, na qual a etiologia é desconhecida ${ }^{(1)}$. Ela pode ser dividida em três formas: aguda/subaguda, esclerosante e granulomatosa(2). A inflamação orbital anterior, inicialmente acomete o globo ocular e os tecidos vizinhos, mas pode, por contigüidade transformar-se numa forma difusa,envolvendo os músculos oculomotores, esclera posterior e nervo óptico ${ }^{(3)}$. Uma vez ins- 
talado o quadro de esclerite posterior, o prognóstico visual torna-se bastante comprometido ${ }^{(4)}$. Apenas trinta por cento dos indivíduos com esclerite posterior apresentam uma doença sistêmica auto-imune associada ${ }^{(5)}$. Em cerca de um terço dos pacientes a doença se manifesta antes dos 40 anos e está associada à esclerite anterior ${ }^{(6-7)}$.

Oitenta e cinco por cento dos casos de esclerite posterior acarretam prejuízo permanente da visão por maculopatia, neuropatia óptica e/ou descolamento de retina exsudativo ${ }^{(8)}$. Em raros casos, a esclerite posterior pode provocar uma reação inflamatória do corpo ciliar, edema dos tecidos adjacentes e evoluir para efusão coroidiana circunferencial levando ao aumento da pressão intra-ocular ${ }^{(9)}$.

\section{RELATO DE CASO}

E.N., 24 anos, sexo masculino, negro, solteiro, residente e natural de Mogi das Cruzes - SP. Apresentou queixa de dor no olho esquerdo (OE) há dez dias de início súbito e que irradiava para o hemicrânio esquerdo. Há cinco dias estava acompanhada de diminuição da acuidade visual, sensação de mal-estar geral, náuseas e vômitos. Na última noite despertou com dor intensa em OE que piorava com a movimentação ocular.

Quanto aos antecedentes oculares, apresentou conjuntivite adenoviral em ambos olhos (AO) há dez meses. De antecedentes sistêmicos vinha apresentando cefaléia e há cinco dias havia sido avaliado pela neurologia, que fez diagnóstico de cefaléia e prescreveu dipirona. De antecedentes familiares: nada digno de nota.

Ao exame oftalmológico apresentava à ectoscopia: proptose e edema na pálpebra superior de OE (Figuras 1A e 1B). Os reflexos pupilares apresentavam-se assimétricos, diminuído à esquerda e ao exame da musculatura extrínseca havia restrição de movimentação de $\mathrm{OE}$ em todas as posições. Acuidade visual (AV) com correção óptica 20/20 e CD 1,5 m para longe e J1 para perto, segundo tabela de Snellen e Jaegger respectivamente. À biomicroscopia apresentava OD sem alterações e em OE hiperemia conjuntival 4+, córnea transparente com precipitados endoteliais finos, câmara anterior rasa com anteriorização do diafragma iridocristaliniano, células e "flare" na câmara anterior $2+$, sinéquia posterior na região temporal inferior e cristalino transparente. Pressão intra-ocular (Po) às 11:00 h de 14 mmHg em OD e 34 mmHg em OE. À gonioscopia em OD: ângulo aberto $360^{\circ}$ visível até esporão escleral e em OE ângulo fechado $360^{\circ}$, não se visibilizando linha de Schwalbe. Ao realizar a indentação corneana observou-se abertura do ângulo até o esporão escleral $360^{\circ}$.O mapeamento de retina e biomicroscopia de fundo de OD mostravam um disco róseo, ovalado, bordas nítidas, escavação $0,3 \times 0,3$, vasos sem alterações, retina aplicada $360^{\circ}$, mácula livre e em OE disco hiperemiado, ovalado, bordas nítidas, escavação $0,3 \times 0,3$, tortuosidade vascular aumentada e edema do pólo posterior (Figura 1C). O teste da fenilefrina a 10\% mostrou-se negativo, confirmando o envolvimento escleral.

Como tratamento inicial, foi administrado em ambiente hospitalar, manitol $250 \mathrm{ml}$ via endovenosa e acetazolamida $250 \mathrm{mg}$ via oral (VO). Após a medicação, a Po foi reduzida para $22 \mathrm{mmHg}$ em OE. Foram prescritas acetazolamida $250 \mathrm{mg}$ 6/6 h e xarope de $\mathrm{KCl} 5 \mathrm{ml}$ 1vez/dia VO e medicação tópica em OE: acetato de prednisolona $1 \% 3 / 3 \mathrm{~h}$, atropina $1 \% 12 / 12 \mathrm{~h}$ e maleato de timolol $0,5 \% 12 / 12 \mathrm{~h}$.

O paciente foi submetido a exames sorológicos que incluíram: PPD: negativo; VDRL: não reagente; FTA-ABS: negativo; fator reumatóide: negativo; IgM para toxo: não reagente; IgG para toxo: 3,96 UI/ml; anti-HIV 1 e 2: Elisa e WesternBlot não reagentes.

A ultra-sonografia ocular (US) mostrou uma rotação anterior do corpo ciliar devido a efusão coroidiana circunferencial, além do espessamento da parede posterior e Tenon retrobulbar $(3,16 \mathrm{~mm})$ e da musculatura extrínseca ocular. A imagem ultra-sonográfica observada é conhecida como "sinal do T" (Figura 1D).

Foram também realizados exames radiológicos: Rx de tórax, bacia e coluna tóraco-lombar sem alterações. Tomografia computadorizada (TC) axial e coronal da órbita revelou importante proptose axial, espessamento difuso da parede ocular, musculatura extrínseca e gordura retro-bulbar (Figura 1E).

O paciente foi encaminhado para avaliação reumatológica e não apresentou sinais de atividade reumática no momento do exame.

Foram realizadas biópsia e exame histopatológico de conjuntiva e glândula lacrimal a fim de se descartar sarcoidose. A biópsia de conjuntiva mostrou epitélio conjuntival e córion com infiltrado inflamatório crônico inespecífico (Figura 2A) e biópsia da glândula lacrimal também revelou infiltrado inflamatório inespecífico entre os ácinos (Figura 2B). Ambas foram coradas pela hematoxilina-eosina.

Por fim, após tais resultados, foi feita a hipótese diagnóstica de inflamação orbitária inespecífica aguda com envolvimento do segmento posterior do globo ocular e a conduta foi a prescrição de prednisona $60 \mathrm{mg} /$ dia $\mathrm{VO}$.

$\mathrm{Na}$ reavaliação após duas semanas de início da corticoterapia sistêmica apresentava-se assintomático com nítida regressão da proptose (Figura 3A). AV c/c: 20/20 AO; biomicroscopia sem alterações em AO, com normalização da profundidade da câmara anterior (CA); Po: $11 \mathrm{mmHg}$ em AO $(14: 00 \mathrm{~h})$; gonioscopia: ângulo aberto $360^{\circ}$ visualizado-se até esporão escleral AO; mapeamento de retina mostrava diminuição da tortuosidade venosa com regressão do edema do pólo posterior e hiperemia do disco óptico (Figura 3B).

A ultra-sonografia ocular mostrou diminuição do espessamento da parede posterior e da musculatura extrínseca ocular. A espessura da parede posterior regrediu de $3,16 \mathrm{~mm}$ para $1,87 \mathrm{~mm}$ (Figura 3C). A TC de órbita revelou diminuição da proptose e do espessamento da parede e musculatura extrínseca. 


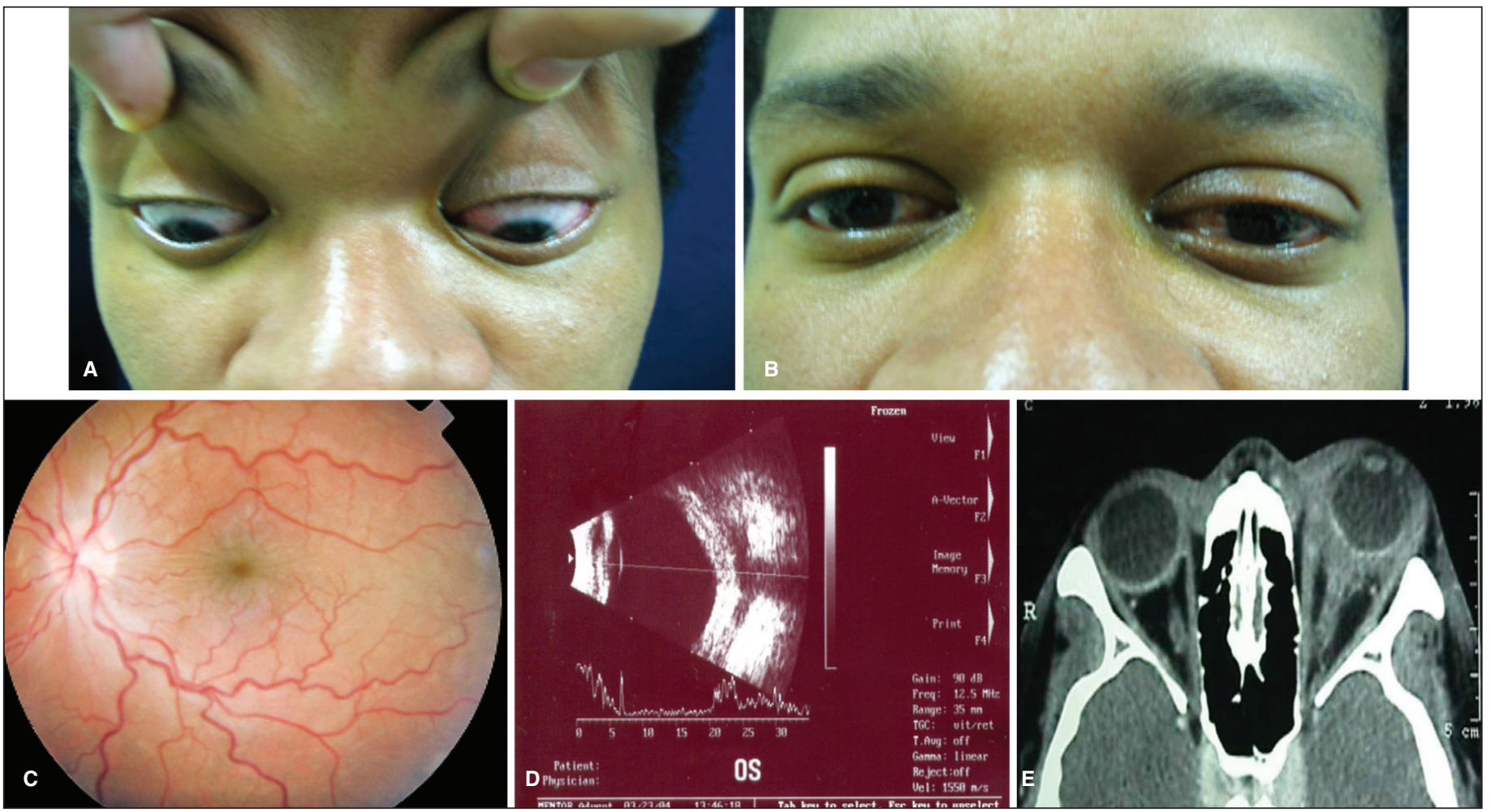

Figura 1 - A e B: quadro inicial do caso, evidenciando proptose de OE; C: retinografia OE mostrando tortuosidade vascular aumentada e edema do pólo posterior; D: US com espessamento da parede ocular $(3,16 \mathrm{~mm})$ e da musculatura extrínseca ocular - "sinal do T"; E: TC órbita revelando proptose axial, espessamento difuso da parede ocular, musculatura extrínseca e gordura retro-bulbar.

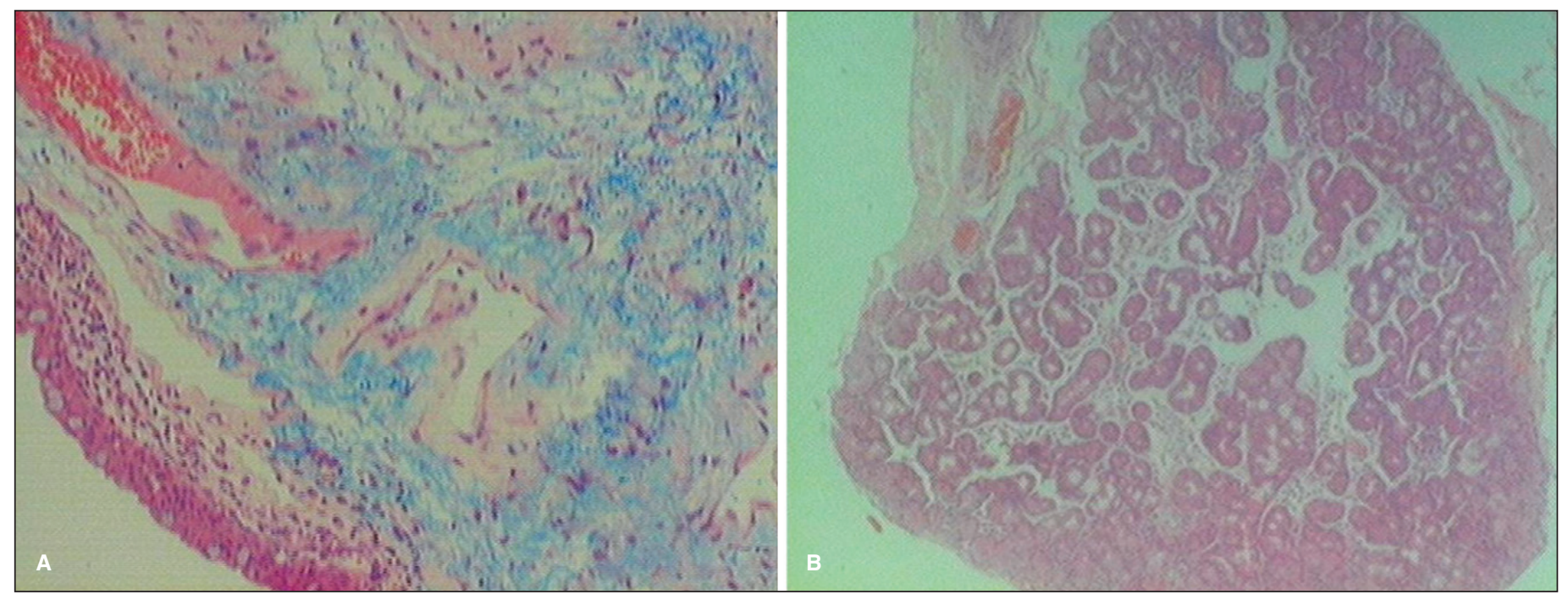

Figura 2 - Exame histopatológico (hematoxilina-eosina) A: epitélio conjuntival e córion com infiltrado inflamatório crônico inespecífico (magnificação x67); B: glândula lacrimal com infiltrado inflamatório crônico inespecífico entre os ácinos (magnificação x235)

\section{DISCUSSÃO}

Por definição, glaucoma secundário ao quadro inflamatório orbitário inespecífico associado a esclerite posterior é um glaucoma pré-trabecular de ângulo fechado sem bloqueio pupilar ${ }^{(9)}$. O fechamento do ângulo ocorre por provável des- locamento anterior da periferia da íris ou de todo o diafragma iridocristaliniano. Mecanismo semelhante ocorre na oclusão da veia central da retina, fotocoagulação panretiniana e retinopexias convencionais que provocam a congestão do corpo ciliar. Massas retro-irianas ou retro-cristalinianas como tumores intra-oculares podem causar o mesmo tipo de glaucoma ${ }^{(10)}$. 


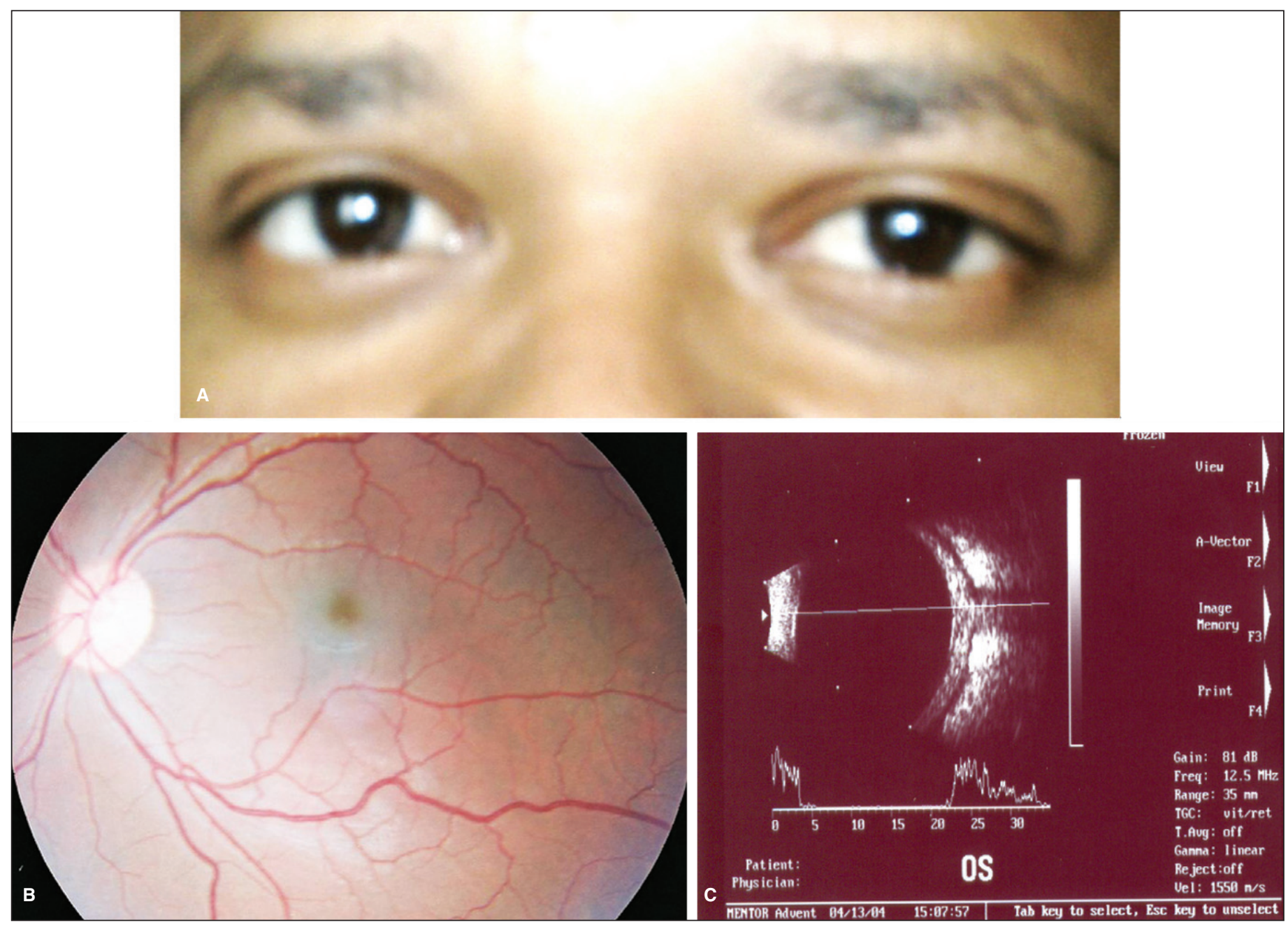

Figura 3 - A: aspecto do paciente após duas semanas de corticoterapia sistêmica com regressão da proptose; B: retinografia com melhora da tortuosidade vascular e edema de pólo posterior; C: US revelando regressão do espessamento da parede posterior (de 3,16 mm para 1,87mm)

A inflamação orbitária inespecífica, na forma difusa, pode provocar uma reação inflamatória do corpo ciliar e edema dos tecidos adjacentes, evoluindo para efusão coroidiana circunferencial. Esta última provoca rotação anterior do corpo ciliar e tem como ponto de apoio o esporão escleral e leva ao fechamento do seio camerular ${ }^{(9)}$. Outro mecanismo provável seria a formação de sinéquias anteriores periféricas secundárias ao processo inflamatório da câmara anterior ${ }^{(10)}$, fato não evidenciado neste relato de caso.

O diagnóstico pode ser facilitado quando há concomitância de esclerite anterior com a posterior (elevação da Po associada a uma câmara rasa e ângulo fechado). Quando o quadro for de esclerite posterior isolada, o diagnóstico etiológico do glaucoma torna-se mais difícil, por isso, frente a um quadro de câmara anterior rasa unilateral, dor e elevação unilateral da Po, deve-se sempre suspeitar de esclerite posterior ${ }^{(8)}$.

O diagnóstico de inflamação orbitária inespecífica difusa é feito por uma anamnese completa através de história e exame oftalmológico, evidenciando dor ao movimento ocular, diminuição da acuidade visual que pode ser melhorada com lentes positivas, proptose, papiledema, dobras de coróide e descolamento de retina exsudativo ${ }^{(8)}$. O US é o principal exame complementar e fornece o diagnóstico de certeza, ao evidenciar o espessamento da parede ocular, Tenon e musculatura extrínsica ${ }^{(11-12)}$.

Uma vez confirmado o diagnóstico de glaucoma de ângulo fechado secundário a um quadro de inflamação orbitária inespecífica, o tratamento deve ser diferente daquele empregado no glaucoma primário de ângulo fechado ${ }^{(13)}$. O tratamento com mióticos é contra-indicado, pois além de não funcionar, leva a uma diminuição da profundidade da câmara anterior agravando o quadro glaucomatoso ${ }^{(14-15)}$. Em contraste, o uso de colírio cicloplégico, neste caso, leva a um aumento da câmara anterior, desfaz o bloqueio pupilar, quando presente, e permite que o ângulo da câmara anterior se abra ${ }^{(13)}$. Se necessário, o uso de colírios hipotensores pode ser usado a fim de reduzir a Po, até que a efusão coroidiana regrida ${ }^{(9)}$. Porém, o tratamento definitivo deve ser dirigido para a causa de todo o processo, a inflamação orbitária.

A terapia consiste na administração de corticóide sis- 
têmico em esquema de pulsoterapia e o uso de indometacina para controle do quadro de dor ${ }^{(16)}$. Com isto, o quadro hipertensivo tende a se resolver dentro de sete dias, em média ${ }^{(13)}$. É importante controlar a Po e prevenir a formação de sinéquias anteriores periféricas, o que torna o glaucoma de difícil controle clinico ${ }^{(17)}$. Quanto ao quadro de uveíte, a etiologia e a presença de doenças sistêmicas devem ser exaustivamente investigadas.

O paciente do presente relato de caso apresentou boa resposta ao tratamento instituído e resolução do quadro glaucomatoso em duas semanas, com restabelecimento da profundidade da CA. Após quatro meses de terapia, apresentou recidiva leve do quadro durante a regressão da corticoterapia sistêmica. No momento, está recebendo dose de manutenção de $5 \mathrm{mg}$ de prednisona por dia para evitar recidivas.

O oftalmologista deve ter sempre em mente que o glaucoma pode estar presente em um quadro de inflamação orbitária. A sua detecção, às vezes, pode ser difícil, pois a hipertensão ocular pode estar mascarada pela exuberância dos sinais flogísticos do segmento anterior do globo ocular ${ }^{(17)}$. É importante reconhecer a causa do glaucoma para que o paciente não seja tratado impropriamente e seja submetido a um procedimento cirúrgico desnecessário ${ }^{18}$.

\section{ABSTRACT}

The nonspecific orbital inflammatory presents several clinical forms. When it evolves the posterior segment of the eye, usually by contiguity, it can lead to serious damage to vision functions. Posterior scleritis causes permanent damage to the vision and rarely progresses to acute glaucoma. Case report: E.N., a 24-year-old black man, complained of pain in the left eye (OS) for ten days, with low visual acuity, malaise, nauseas and vomiting. On ophthalmologic examination, he presented proptosis, restricted eye movements and edema on the upper left eyelid. Best-corrected visual acuity was $20 / 20$ in OD and counting fingers at $1.5 \mathrm{~m}$ in OS. The intraocular pressure was $14 \mathrm{mmHg}$ in OD and $34 \mathrm{mmHg}$ in OS. The biomicroscopy presented in OS conjunctival hyperemia cornea with keratic precipitates, shallow anterior chamber with cells and flare 2+. Gonioscopy in OS showed angleclosure of $360^{\circ}$. The ophthalmoscopic examination revealed increased vascular tortuosity and posterior pole edema. Treatment for acute glaucoma was initiated and complementary tests were ordered. Ocular ultrasonography and orbit computerized tomography showed a diffuse thickening of the ocular wall and extrinsic muscles. Other tests were normal. The presumptive diagnosis was acute nonspecific orbital inflammation affecting the ocular bulb posterior segment together with acute glaucoma. He initiated on prednisone $60 \mathrm{mg} /$ day PO. After two weeks of systemic corticotherapy, the patient was asymptomatic, with evident regression of proptosis and scleritis and normal intraocular pressure (11 $\mathrm{mmHg}$ in $\mathrm{AU})$. Although not very frequent, acute glaucoma may be present in orbital inflammatory process and should be treated with systemic corticotherapy and topical medication.

Keywords: Orbital diseases/complications; Scleritis; Glaucoma, angle-closure/etiology; case reports [Publication type]

\section{REFERÊNCIAS}

1. Dantas AM, Monteiro MLR. Doenças da órbita. Rio de Janeiro: Cultura Médica; 2002.

2. Rootman J, Nugent R. The classification and management of acute orbital pseudotumors. Ophthalmology. 1982;89(9):1040-8.

3. Dolman PJ, Rootman J. Nonspecific orbital inflammatory syndromes. In: Fraunfelder FT, Roy FH, editors. Current ocular therapy. Philadelphia: Saunders; 2000. p.574-6.

4. Buenestado Marjalizo F, Redondo Garcia I, Bonales Daimiel J, del Rio Fernandez S. Posterior scleritis with exudative retinal detachment. Arch Soc Esp Oftalmol. 2000;75(9):643-6. Spanish.

5. Wang JK, Lai PC, Yang CH. Subretinal mass as a presenting sign of posterior scleritis: a case report. Kaohsiung J Med Sci. 2003;19(10):522-5.

6. McCluskey PJ, Watson PG, Lightman S, Haybittle J, Restori M, Branley M. Posterior scleritis: clinical features, systemic associations, and outcome in a large series of patients. Ophthalmology. 1999;106(12):2380-6.

7. Jabs DA, Mudun A, Dunn JP, Marsh MJ. Episcleritis and scleritis: clinical features and treatment results. Am J Ophthalmol. 2000;130(4):469-76.

8. Oréfice F, organizador. Uveíte, clínica e cirúrgica. Rio de Janeiro: Cultura Médica; 2000.

9. Almeida HG, Almeida GV, Calixto N, Carvalho CA, editores. Glaucomas secundários. São Paulo: Roca; 1989.

10. Wilhelmus KR, Grierson I, Watson PG. Histopathologic and clinical associations of scleritis and glaucoma. Am J Ophthalmol. 1981;91(6):697-705.

11. Baráková D, Bujalková D, Redinová M. [Echographic findings in posterior scleritis]. Cesk Slov Oftalmol. 2003;59(2):86-90. Czech.

12. Biswas J, Mittal S, Ganesh SK, Shetty NS, Gopal L. Posterior scleritis: clinical profile and imaging characteristics. Indian J Ophthalmol. 1998;46(4): 195-202.

13. Quinlan MP, Hitchings RA. Angle-closure glaucoma secondary to posterior scleritis. Br J Ophthalmol. 1978;62(5):330-5.

14. Mapstone R. Mechanisms in open-angle glaucoma. Br J Ophthalmol. 1978; 62(5):275-82.

15. Poinoosawmy D, Nagasubramanian S, Brown NA. Effect of pilocarpine on visual acuity and on the dimensions of the cornea and anterior chamber. $\mathrm{Br} \mathrm{J}$ Ophthalmol. 1976;60(10):676-9.

16. Heiligenhaus A, Dück N, Michel D, Hudde T, Koch J, Steuhl KP. [Indication and effect of immunosuppression in patients with scleritis]. Klin Monatsbl Augenheilkd. 2003;220(7):471-80. German.

17. Watson PG. Glaucoma associated with keratitis, episcleritis and scleritis. In: Ritch R, Shields MB, Krupin T. The glaucomas: clinical science. 2nd ed. St. Louis: Mosby; c1996. v.2. cap.57. p.1207-23.

18. Phelps CD. Angle-closure glaucoma secondary to ciliary body swelling. Arch Ophthalmol. 1974;92(4):287-90 